\title{
Scalable Feedback Control for Distributed Beamforming in Sensor Networks
}

\author{
R. Mudumbai, J. Hespanha, U. Madhow and G. Barriac \\ Dept. of Electrical and Computer Engineering \\ University of California, Santa Barbara, CA 93106 \\ Email:raghu@ece.ucsb.edu, hespanha@ece.ucsb.edu,madhow@ece.ucsb.edu, barriac@engineering.ucsb.edu
}

\begin{abstract}
Recent work has shown that large gains in communication capacity are achievable by distributed beamforming in sensor networks. The principal challenge in realizing these gains in practice, is in synchronizing the carrier signal of individual sensors in such a way that they combine coherently at the intended receiver. In this paper, we provide a scalable mechanism for achieving phase synchronization in completely distributed fashion, based only on feedback regarding the power of the net received signal. Insight into the workings of the protocol is obtained from a simple theoretical model that provides accurate performance estimates.
\end{abstract}

\section{INTRODUCTION}

Recently there has been significant interest in the communication problem in sensor networks. While several interesting communication models have been proposed and analyzed in the literature [1], [2], we concentrate here on the model proposed in [3]. The basic assumption of this model is the availability of redundant, spatially-distributed, powerconstrained nodes that can cooperatively transmit a common message signal to a remote station. The goal is to exploit the spatial diversity and redundancy available in the sensor network to achieve energy efficient communication. The challenge is in devising protocols to coordinate the transmissions of individual sensors in a distributed fashion.

In the distributed beamforming system considered in [3], the individual sensors adjust their transmissions such that they combine coherently (in-phase) at the receiver. This requires that the sensors be able to measure or estimate the channel accurately, and that the sensors share a common phase reference. While channel-estimation can be performed by reciprocity using pilot symbols transmitted by the intended receiver, establishing a common phase reference is an important practical challenge. This is because small timing differences between sensors, e.g. from channel propagation delays, can still cause large phase offsets with high frequency carriers. In [3], a master-slave architecture was proposed for this purpose, where slave sensors derive their phase reference from a designated master sensor, while correcting for propagation delay lags to the master sensor. In this paper, we propose an alternative

This work was supported by the National Science Foundation under grants CCF-0431205, ANI-0220118 and EIA-0080134, by the Office of Naval Research under grant N00014-03-1-0090, and by the Institute for Collaborative Biotechnologies through grant DAAD19-03-D-0004 from the U.S. Army Research Office. approach, in which feedback from the receiver is employed by each sensor to adjust its phase.

We consider the following model to illustrate our ideas. The protocol is initialized by each sensor transmitting a common message signal modulated by a carrier with an arbitary phase offset. (This phase offset is a result of timing synchronization errors.) When the sensors' wireless channel is linear and timeinvariant, the received signal is the message signal modulated by an effective carrier signal that is the phasor sum of the channel-attenuated carrier signals of the individual sensors. At periodic intervals, the receiver broadcasts a feedback message to the sensors conveying the received SNR level of the preceding transmission. Each sensor introduces an independent random perturbation of their transmitted phase offset. When this results in increased SNR compared to the previous timeslot, the new phase offset is set equal to the perturbed phase by each sensor; otherwise, the new phase offset is set equal to the phase prior to the perturbation. Each sensor then introduces a new random perturbation, and the process continues. We show that this procedure asymptotically achieves a phase synchronized system, and provide an analysis that accurately predicts the rate of convergence.

Related Work: Much of the focus of the growing literature on cooperative transmission has been on obtaining diversity gains. The authors in [4] first looked at the problem of cooperative transmission in the context of cellular mobiles. They showed that diversity gains can be realized over single node transmission. Multiple cooperating relays between a source and destination are considered in [5], where it is shown that distributed coding, along with amplify-and-forward or decodeand-forward strategies achieves maximum diversity gain. In these works, the cooperating mobiles are not assumed to have carrier synchronization, and they transmit on orthogonal subchannels. If we fix the power per transmitter, such strategies can achieve gains in range because the powers add at the receiver, and because of the diversity obtained for combating impairments such as fading and shadowing. In contrast, our objective is to obtain beamforming gains, which require the coherent addition of the amplitudes of multiple transmitters, which allow reduction of the power per transmitter for a given range. Distributed beamforming requires much more stringent synchronization across participating nodes than distributed diversity, although we have shown recently [6] that 
synchronization can also be a limiting factor for some classes of distributed diversity schemes.

There is also some recent work on distributed beamforming in the literature. It is pointed out in [7] that distributed beamforming relays offers the possibility of achieving power efficiency in a wireless ad-hoc network, they also show that even partial phase synchronization leads to significant increase in network performance. In our own prior work [3], we consider distributed beamforming in sensor networks using a master-slave architecture for a cluster of cooperating nodes. The degradation due to synchronization errors was examined, and detailed methods for achieving synchronization in practice were presented. The directivity patterns achieved through random beamforming are studied in [8]. Using a physical propagation path model, it is shown that the "average beampattern" achieves a directivity that scales linearly with number of sensors, which are assumed to be randomly located on a surface area. However, carrier phase synchronization across sensors is not addressed in [8]. To the best of our knowledge, this is the first paper to consider feedback control for distributed carrier synchronization in sensor networks.

Outline: Section II describes the communication model and the assumptions used in the paper. Section III formulates a distributed feedback control protocol for phase synchronization. In Section IV, we present a theoretical analysis for the synchronization protocol and numerical results in Section $\mathrm{V}$ validate the analysis. Section VI suggests possible extensions of this work and concludes the paper.

\section{SySTEM MODEL}

We consider a system of $N$ sensors transmitting a common message signal $m(t)$ to a receiver. The sensors are power constrained to a maximum transmit power of $P$. The message $m(t)$ could represent raw measurement data, or it could be a waveform encoded with digital data. We now list the assumptions in this model.

1) The sensors communicate with the receiver over a narrowband wireless channel at some carrier frequency, $f_{c}$. In particular, the message bandwidth $B<W_{c}$, where $B$ is the bandwidth of $m(t)$ and $W_{c}$ is the coherence bandwidth of each sensor's channel. In other words, each sensor is assumed to have a flat-fading channel to the receiver. Therefore the sensor $i$ 's channel can be represented by a complex scalar gain $h_{i}$.

2) The sensors' communication channel is time-slotted with slot length $T$. The sensors only transmit at the beginning of a slot. This assumes that $\tau_{\max } B \ll 1$, where $\tau_{\max }$ is an upper bound on sensor timing errors. In other words, timing errors are small compared to a symbol interval (a "symbol interval" $T_{s}$ is nominally defined as inverse bandwidth: $T_{s}=\frac{1}{B}$ ).

3) Each sensor has a local oscillator synchronized to the carrier frequency $f_{c}$ i.e. carrier drift is small. One way to ensure this is to use Phase-Locked Loops (PLLs) to synchronize to a reference tone transmitted by a designated master sensor as in [3]. This allows us to use complex-baseband notation for all the transmitted signals referred to the carrier frequency $f_{c}$.

4) The local carrier of each sensor $i$ has an unknown phase offset, $\gamma_{i}$ relative to the receiver's phase reference. Note that even using PLLs for synchronization, still results in independent random phase offsets $\gamma_{i}=\left(2 \pi f_{c} \tau_{i}\right.$ $\bmod 2 \pi)$, because of timing synchronization errors $\tau_{i}$ that are fundamentally limited by propagation delay effects.

5) The channels $h_{i}$ are assumed to exhibit slow-fading, i.e. the channel gains stay roughly constant for several timeslots. In other words $T_{s} \ll T \ll T_{c}$, where $T_{c}$ is the coherence time of the sensor channels.

Distributed transmission model: The communication process begins with the receiver broadcasting a signal to the sensors to transmit their measured data. The sensors then transmit the message signal at the next time-slot. Specifically, each sensor transmits: $s_{i}(t)=A \cdot g_{i} m\left(t-\tau_{i}\right)$, where $\tau_{i} \leq \tau_{\max }$ is the timing error of sensor $i, A=\sqrt{P}$ is the amplitude of the transmission, and $g_{i}$ is a complex amplification performed by sensor $i$. Our objective is to choose $g_{i}$ to achieve optimum received SNR, and the scaling factor $A$ represents the transmit power constraint. For simplicity, we write $h_{i}=a_{i} e^{j \psi_{i}}$ and $g_{i}=b_{i} e^{j \theta_{i}}$. Then the received signal is:

$$
\begin{aligned}
r(t) & =\sum_{i=1}^{N} h_{i} s_{i}(t) e^{j \gamma_{i}}+n(t) \\
& =A \sum_{i=1}^{N} h_{i} g_{i} e^{j \gamma_{i}} m\left(t-\tau_{i}\right)+n(t) \\
& =A \sum_{i=1}^{N} a_{i} b_{i} e^{j\left(\gamma_{i}+\theta_{i}+\psi_{i}\right)} m\left(t-\tau_{i}\right)+n(t) .
\end{aligned}
$$

In the frequency domain, this becomes:

$$
\begin{aligned}
R(f) & =A \sum_{i=1}^{N} a_{i} b_{i} e^{j\left(\gamma_{i}+\theta_{i}+\psi_{i}\right)} M(f) e^{-j f \tau_{i}}+N(f) \\
& \approx A \cdot M(f) \sum_{i=1}^{N} a_{i} b_{i} e^{j\left(\gamma_{i}+\theta_{i}+\psi_{i}\right)}+N(f),
\end{aligned}
$$

where $n(t)$ is the additive noise at receiver and $N(f)$ is its Fourier transform over $|f|<\frac{B}{2}$.

In (1), the phase term $\gamma_{i}$ accounts for the phase offset in sensor $i$. In (3), we set $e^{-j f \tau_{i}} \approx 1$ because $B \tau_{i} \ll 1$. Equation (3) motivates a figure of merit for the beamforming gain:

$$
G=\frac{\left|\sum_{i=1}^{N} a_{i} b_{i} e^{j\left(\gamma_{i}+\theta_{i}+\psi_{i}\right)}\right|^{2}}{\sum_{i=1}^{N}\left|b_{i}\right|^{2}},
$$

where $G$ is the ratio of the received power to total transmit power.

Note that $b_{i} \leq 1$, in order to satisfy the power constraint on sensor $i$. From the Cauchy-Schwartz Inequality, we can see that to maximize $G$, we require:

$$
\gamma_{i}+\theta_{i}^{o p t}+\psi_{i}=C
$$


where $C$ is a constant. However sensor $i$ is unable to estimate either $\gamma_{i}$ or $\psi_{i}$ because of the lack of a common carrier phase reference. In the rest of this paper, we propose feedback control methods for sensor $i$ to dynamically compute $\theta_{i}^{o p t}$ so as to achieve (5).

\section{FeedBack Control Protocol}

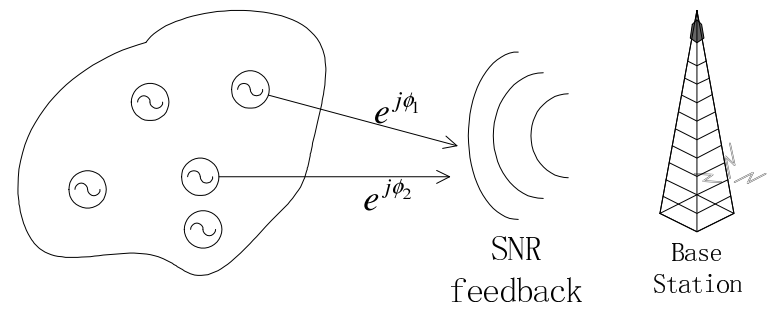

Fig. 1. Phase synchronization using receiver feedback

Figure 1 illustrates the process of phase synchronization using feedback control. The protocol works as follows: each sensor starts with an arbitrary unsynchronized phase offset. In each time-slot, the sensor applies a random phase perturbation and observes the resulting received signal strength $y[n]$ through feedback. The objective is to adjust its phase to maximize $y[n]$ through coherent combining at the receiver. Each phase perturbation is a guess by each sensor about the correct phase adjustment required to increase the overall received signal strength. If the received SNR is found to increase as a result of this perturbation, the sensor adds the appropriate phase offset, and repeats the process. This works like a distributed, randomized gradient search procedure, and eventually converges to the correct phase offsets for each sensor to achieve distributed beamforming. Figure 2 shows the convergence to received beamforming with $N=10$ sensors.

Let $n$ denote the time-slot index and $y[n]$ the amplitude of the received signal in time-slot $n$. We have: $y[n] \propto$ $\left|\sum_{i} a_{i} b_{i} e^{j \phi_{i}[n]}\right|$ where $\phi_{i}=\gamma_{i}+\theta_{i}+\psi_{i}$ is the received phase, and all the coefficients $a_{i}, b_{i}$, and the proportionality constant are taken to be unity (for simplicity of analysis).

We set the initial phases $\theta_{i}[0]=0$. At each time-slot, the sensor $i$ observes the feedback $y[n]$ (assumed noiseless) and applies a perturbation to its phase for the next time-slot: $\theta_{i}[n+$ $1]=\theta_{i}[n]+u_{i}[n]+\delta_{i}[n]$, which results in a similar perturbation in received phase: $\phi_{i}[n+1]=\phi_{i}[n]+u_{i}[n]+\delta_{i}[n]$. The perturbation consists of two components: an adaptive control component $u_{i}[n]$ and a random component $\delta_{i}[n]$. The random component is simply set to $\delta_{i}[n]= \pm \delta$ with equal probability where $\delta$ is a parameter of the protocol, independently across sensors and across time-slots. The adaptive component is set according to:

$$
u_{i}[n]= \begin{cases}\delta_{i}[n-1] & y[n]>y[n-1] \\ 0 & \text { otherwise }\end{cases}
$$
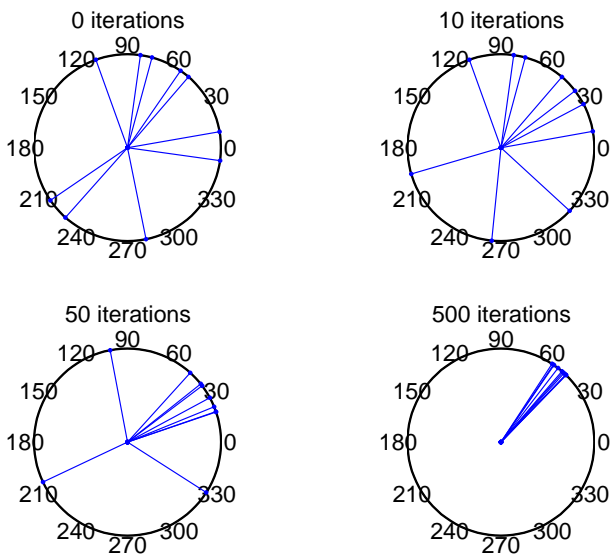

Fig. 2. Convergence of distributed beamforming algorithm

\section{Convergence Analysis}

We first show that the objective function of total received SNR has a unique global maximum, and then provide an analysis of the rate of convergence that closely matches simulations.

\section{A. Globally Optimum Phase Offsets}

Consider $f(\bar{\phi}) \doteq f\left(\phi_{1}, \phi_{2}, . . \phi_{N}\right)=\left|\sum_{i=1}^{N} e^{j \phi_{i}}\right|^{2}$. The problem of achieving distributed beamforming is the same as the problem of maximizing $f(\bar{\phi})$. We now look for the critical points of this function. We have:

$$
\begin{aligned}
f(\bar{\phi}) & =\left(\sum_{i=1}^{N} e^{j \phi_{i}}\right) \cdot\left(\sum_{j=1}^{N} e^{j \phi_{j}}\right)^{*} \\
& =N+\sum_{i \neq j, i, j=1}^{N} e^{j\left(\phi_{i}-\phi_{j}\right)} .
\end{aligned}
$$

We obtain the following expression for the partial derivatives:

$$
\frac{\partial f}{\partial \phi_{k}}=-\sum_{j \neq k, j=1}^{N} \sin \left(\phi_{j}-\phi_{k}\right) .
$$

To find the critical points of the function $f(\bar{\phi})$, we set the RHS of (7) to zero for all $k$. The only way to satisfy the resulting set of equation is: $\phi_{i}-\phi_{j}=m \pi, \forall i, j$. If we express the phases $\phi_{i}$ to be modulo $2 \pi$, then we can simplify this condition to $\phi_{i}=C$ or $\phi_{i}=C+\pi, \forall i=1 . . N$ for some constant $C$. We can demonstrate (details omitted) that a critical point of $f(\bar{\phi})$ is a saddle point unless $\phi_{i}=C, \forall i$ in which case it is a local (and global) maximum. We therefore conclude that any gradient search procedure converges to a global maximum of $f(\bar{\phi})$, and hence achieves distributed beamforming. However, it is desirable to have a theoretical model that gives some insight into the rate of convergence of the received SNR, and its dependence on the random perturbation parameter $\delta$. This analysis is carried out in the next section. 


\section{B. Rate of Convergence}

We now present an analytical model that predicts the convergence behavior of the synchronization protocol presented in Section III. In this protocol, the control applied by the sensors (as in (6)) is highly non-linear, therefore exact analysis is difficult. We present a theoretical model that is based on a series of approximate arguments, and show empirically that it gives accurate results.

For the analytical model, let $y[n]$ represent the received signal strength in the $n$ 'th time-slot, which is also known to the sensors as noiseless feedback at the end of the time-slot. (This is consistent with the notation in Section III.) We want to model the increase in signal strength $\Delta y$ as a result of the random phase perturbation by the sensors. This is a random variable that can be written as follows:

$$
\begin{aligned}
h(y[n], \bar{\delta}[n]) & \doteq \Delta y[n] \\
& = \begin{cases}y[n+1]-y[n] & y[n+1]>y[n] \\
0 & \text { otherwise, }\end{cases}
\end{aligned}
$$

where $\bar{\delta}[n] \equiv\left[\delta_{1}[n], \delta_{2}[n], \ldots \delta_{N}[n]\right]$.

Consider a sequence of $M$ time-slots. The total increase in received signal strength over the $M$ time-slots is:

$$
\begin{aligned}
\Delta_{M} y[n] & =\sum_{k=0}^{M-1} h(y[n+k], \bar{\delta}[n+k]) \\
& \approx \sum_{k=0}^{M-1} h(y[n], \bar{\delta}[n+k]) \\
& \approx M \cdot E_{\bar{\delta}}[h(y, \bar{\delta})] .
\end{aligned}
$$

Equation (9) is based on the assumption that the phase perturbation $\delta$ is small enough that the signal strength variation is small in $M$ time-slots. Equation (10) uses the fact that $\delta_{i}[n]$ is chosen iid across time-slots and therefore the law of large numbers applies. Essentially we want to replace the random signal strength variations by the ensemble average variation for the purposes of the theoretical analysis. These approximations require that $M$ is large enough for the law of large numbers to apply, and $\delta$ is small enough that $E_{\bar{\delta}[n]}[h(y[n], \bar{\delta}[n])]$ is roughly constant over $M$ time-slots. Rather than trying to justify these assumptions, we show that it leads to accurate results for reasonable choices of the parameter $\delta$.

Next we obtain expressions for the ensemble average signal strength increase per time-slot:

$$
\frac{\Delta_{M} y[n]}{M} \approx E_{\bar{\delta}}[h(y, \bar{\delta})]=E_{\bar{\delta}}[\max (0, \bar{h})]
$$

where

$$
\begin{aligned}
\bar{h} & \doteq\left|\sum_{i=1}^{N} e^{j\left(\phi_{i}+\delta_{i}\right)}\right|-\left|\sum_{i=1}^{N} e^{j \phi_{i}}\right| \\
& =\left|y \cos \delta-\sum_{i=1}^{N} \sin \phi_{i} \sin \delta_{i}+j \sum_{i=1}^{N} \cos \phi_{i} \sin \delta_{i}\right|-y \\
& =\left|y \cos \delta+x_{1}+j x_{2}\right|-y
\end{aligned}
$$

with $x_{1}, x_{2}$ defined as the real and imaginary parts of the random terms in (11); this is shown in Figure 3.

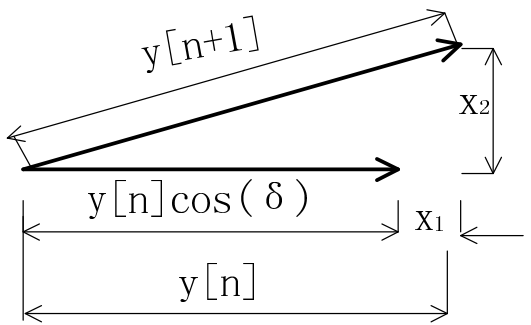

Fig. 3. In-phase and quadrature components of random signal increments

Since $\delta_{i}$ are iid across sensors, it follows that $\sin \delta_{i}$ are also iid, and by using the Central Limit Theorem for large $N, x_{1}, x_{2}$ can be regarded as zero-mean Gaussian random variables. Furthermore from (11), it can be shown that $\sigma_{1}^{2}+$ $\sigma_{2}^{2}=N \cdot \sin ^{2} \delta$, where $\sigma_{1}, \sigma_{2}$ are the variances of $x_{1}, x_{2}$ respectively. The exact values of $\sigma_{1}, \sigma_{2}$ depend on the phase offsets $\phi_{i}$. The $\phi_{i}$ need to be clustered together in a range $\phi_{i} \sim\left(-\phi_{0}, \phi_{0}\right)$, that depends on the instantaneous value of $y[n]$. We have the following estimates for the variances:

$$
\begin{aligned}
\sigma_{1}^{2} & \approx \frac{N}{2} \cdot \sin ^{2} \delta \cdot\left(1+\frac{y}{N} \cos \left(\frac{\pi y}{N}\right)\right) \\
\sigma_{2}^{2} & \approx \frac{N}{2} \cdot \sin ^{2} \delta \cdot\left(1-\frac{y}{N} \cos \left(\frac{\pi y}{N}\right)\right) .
\end{aligned}
$$

To see this, we model the $\phi_{i}$ as being distributed uniformly between $\pm \phi_{0}$. (The $\phi=0$ reference can be chosen such that the distribution is symmetric.) Using the condition $N . E_{\phi_{i}}\left[\cos \phi_{i}\right]=y$, it follows that $\frac{\sin \phi_{0}}{\phi_{0}}=\frac{y}{N}$. Then

$$
\begin{aligned}
\sigma_{1}^{2} & \equiv \sin ^{2} \delta \sum_{i=1}^{N} \sin ^{2} \phi_{i} \\
& \approx N \cdot \sin ^{2} \delta \cdot E_{\phi_{i}}\left[\sin ^{2} \phi_{i}\right] \\
& =\frac{N}{2} \sin ^{2} \delta\left(1-E_{\phi_{i}}\left[\cos \left(2 \phi_{i}\right)\right]\right),
\end{aligned}
$$

where

$$
\begin{aligned}
E_{\phi_{i}}\left[\cos \left(2 \phi_{i}\right)\right] & =\frac{\sin \left(2 \phi_{0}\right)}{\left(2 \phi_{0}\right)}=\frac{y}{N} \cos \phi_{0} \\
& \approx-\frac{y}{N} \cos \left(\frac{\pi y}{N}\right) .
\end{aligned}
$$

Note in (13), we invoked the law of large numbers for large $N$. Using (11) and (12), we can now compute an analytical estimate for $\bar{h}$, the ensemble average of increase of received signal strength in one time-slot:

$$
\begin{aligned}
\frac{\Delta_{M} y[n]}{M}= & E[\bar{h} \mid \bar{h} \geq 0] P(\bar{h} \geq 0) \\
\approx & P\left(x_{1}>y(1-\cos \delta)\right) E\left[\bar{h} \mid x_{1}>y(1-\cos \delta)\right] \\
& +P\left(\left|x_{2}\right|>y \sin \delta\right) E\left[\bar{h}|| x_{2} \mid>y \sin \delta\right]
\end{aligned}
$$

where we used:

$$
\{\bar{h} \geq 0\} \approx\left\{x_{1}>y(1-\cos (\delta))\right\} \cup\left\{\left|x_{2}\right|>y \sin \delta\right\} .
$$


Equation (15) can be readily computed for Gaussian random variables $x_{1}, x_{2}$, and is the basis for the results presented in Section V.

\section{NumericAl RESUlts}

We now present some simulation results to demonstrate the feedback control protocol presented in Section III. Figure 4 shows the asymptotic convergence of the received signal amplitude to the maximum (corresponding to distributed beamforming), for the case of $N=100$ sensors. As expected, the received signal strength grows rapidly in the beginning, and as a rule of thumb it takes about $K=5 N$ time-slots to achieve signal amplitudes of about 0.75 of the maximum. It can be seen that the analytical results based on (15) match simulated results based on (6) very accurately. The advantage of the analytical approach is that the resulting model of the convergence behavior of the system is deterministic and smooth compared to the simulation results.

There is also a tradeoff between the initial convergence rate and long-term performance of the protocol, that depends on the choice of parameter $\delta$. Figure 6 shows this tradeoff for 100 sensors and 500 timeslots. We observe that the system performs reasonably well, over a range of $\delta$ values. Again the analytical model captures the tradeoff very accurately. Finally Figure 5 shows the variation of the "beamforming gain" with number of sensors for a fixed number of time-slots.

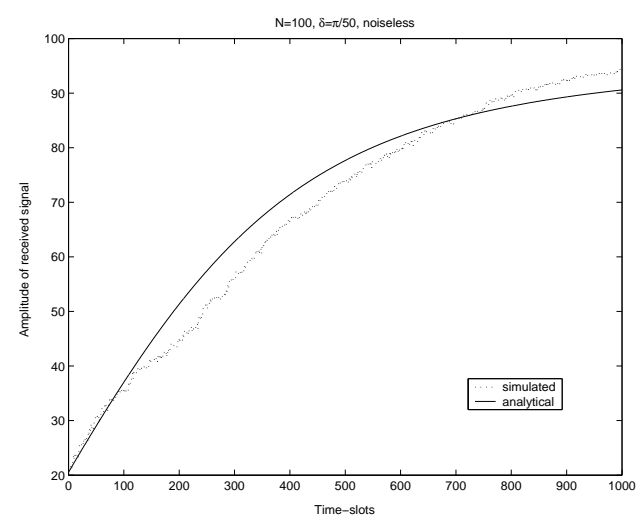

Fig. 4. Phase synchronization with noiseless feedback: $N=100$

\section{CONCLUSION}

The proposed distributed beamforming scheme scales to very large numbers of sensors because of two features: first, the feedback is not specialized to each sensor, but is simply the received signal power (the quantity to be maximized); second, the sensors' actions in response to the feedback are completely uncoordinated. Given the excellent agreement between analysis and simulations, we have detailed insight into the workings of the algorithm: about $40 \%$ of the achievable beamforming gain is attained very rapidly, followed by a slower convergence to an asymptotic value. These promising preliminary results motivate a number of directions for future research. Practical aspects to be explored include analyzing

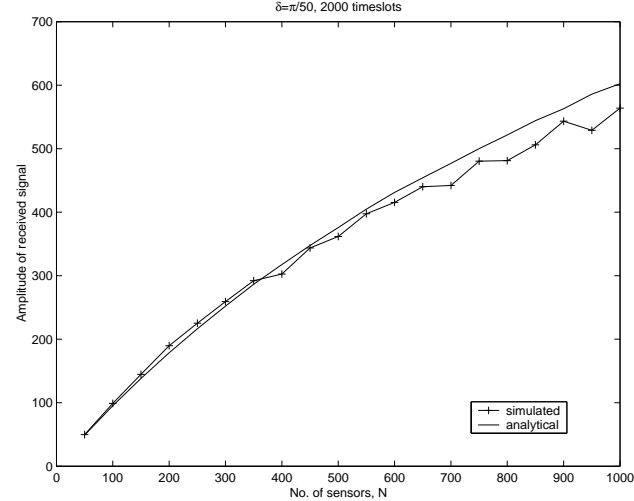

Fig. 5. Received signal strength vs number of sensors

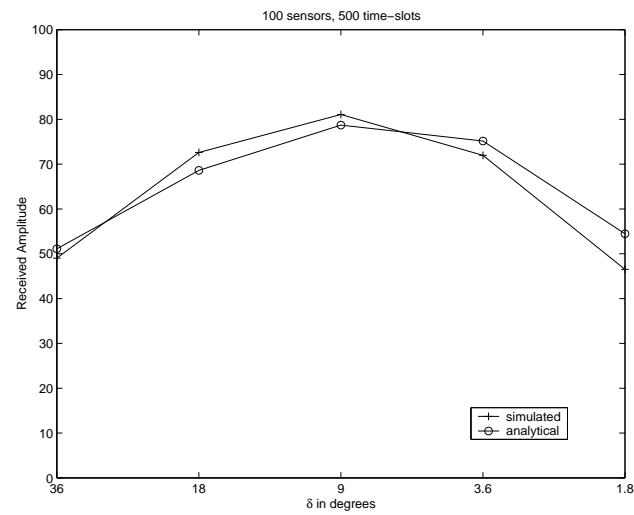

Fig. 6. Received signal strength vs perturbation parameter $\delta$

the the effects of noise and the ability of the feedback control algorithm to track a time-varying channel.

\section{REFERENCES}

[1] B. Ananthasubramaniam and U. Madhow, "Virtual radar imaging for sensor networks," in Proc. 3rd International Symposium on Information Processing in Sensor Networks (IPSN'04), pp. 294-300, Apr. 26-27, 2004.

[2] J. Gomez, A. T. Campbell, M. Naghshineh, and C. Bisdikian, "Poweraware routing in wireless packet networks," in Proc. 1999 IEEE International Workshop on Mobile Multimedia Communications (MOMUC'99), pp. 380-383, Nov. 15-17, 1999.

[3] G. Barriac, R. Mudumbai, and U. Madhow, "Distributed beamforming for information transfer in sensor networks," in Proc. 3rd International Symposium on Information Processing in Sensor Networks (IPSN'04), pp. 81-88, Apr. 26-27, 2004.

[4] A. Sendonaris, E. Erkip, and B. Aazhang, "User cooperation diversity. part i. system description," vol. 51, pp. 1927-1938, Nov. 2003.

[5] J. Laneman and G. Wornell, "Distributed space-time-coded protocols for exploiting cooperative diversity in wireless networks," vol. 49, pp. 24152425, Oct. 2003.

[6] R. Mudumbai, G. Barriac, and U. Madhow, "Spread-spectrum techniques for distributed space-time communication in sensor networks," in Proc. 38th Asilomar Conference on Signals, Systems and Computers (Asilomar'04), (Pacific Grove, CA), Nov. 7-10, 2004.

[7] B. Hassibi and A. F. Dana, "On the power efficiency of sensory and ad-hoc wireless networks," preprint, submitted to .

[8] H. Ochiai, P. Mitran, H. V. Poor, and V. Tarokh, "Collaborative beamforming in ad hoc networks," in Proc. 2004 IEEE Inform. Theory Workshop, Oct. 24-29, 2004. 